\title{
An infinite-horizon maximum principle with bounds on the adjoint variable
}

\author{
Thomas A. Weber* \\ Department of Management Science and Engineering, Terman Engineering Center, \\ Stanford University, Stanford, CA 94305-4026, USA \\ Received 11 June 2002; accepted 23 November 2004 \\ Available online 25 February 2005
}

\begin{abstract}
We provide necessary optimality conditions for a general class of discounted infinitehorizon dynamic optimization problems. As part of the resulting maximum principle we obtain explicit bounds on the adjoint variable, stronger than the transversality conditions in Arrow-Kurz form.
\end{abstract}

(C) 2005 Elsevier B.V. All rights reserved.

JEL classification: $\mathrm{C} 60 ; \mathrm{C} 61$

Keywords: Dynamic optimization; Infinite-horizon optimal control; Necessary optimality conditions

\section{Introduction}

Infinite-horizon dynamic optimization problems typically have an incomplete set of necessary optimality conditions that do not allow the effective selection of good candidates for an optimal solution. The maximum principle by Pontryagin et al. (1962) has been extended to infinite-horizon problems by Arrow and Kurz (1970) as

\footnotetext{
*Tel.: + 16507256827 ; fax: + 16507231614 .

E-mail address: webert@stanford.edu.
} 
well as Halkin (1974), but transversality conditions (i.e., additional conditions on the adjoint variable) are generally not available. ${ }^{1}$ In fact, Halkin (1974) provides a counterexample for a 'natural' extension of the finite-horizon transversality conditions proposed by Arrow and Kurz. ${ }^{2}$ Thus, some care is necessary when formulating strong necessary conditions for infinite-horizon optimal control problems. Using the method of smooth approximation (reviewed by Aseev, 1999), it is possible to obtain a weak transversality condition in the form of 'asymptotic stationarity' of the (maximized) Hamiltonian, ${ }^{3}$ and positivity of the adjoint variable (Aseev et al., 2001a, b). Here, instead of imposing growth limitations and monotonicity on state trajectories, we modify the results by Aseev et al. to suit the common situation, where the state space is a compact invariant set. Invariance of the state space is satisfied in many economic applications whenever finite economic resources impose limits on the magnitude of the state variables. Under this natural invariance condition, together with a number of technical assumptions, we are able to obtain bounds on the adjoint variable (i.e., growth conditions), stronger than the aforementioned Arrow-Kurz 'natural' transversality conditions for a general class of systems. Such bounds can be used to show asymptotic convergence of optimal trajectories (cf. our example in Section 5) and to approximate optimal solutions via forward simulation of the Hamiltonian system (Lee and Markus, 1967; Weber, 2001).

\section{Statement of the problem}

Let us consider the system

$$
\dot{x}=F(x) u+g(x) \text {, }
$$

where $F=\operatorname{diag}\left(f_{1}, \ldots, f_{n}\right)^{\prime}$ and $\left(g, f_{i}\right): X \rightarrow\left(\mathbb{R}^{n}, \mathbb{R}\right)$ are continuously differentiable ${ }^{4}$ functions for $i=1,2, \ldots, n$, with $X \subset \mathbb{R}^{n}$, and $n$ is a positive integer. Let $U=$ $\left[\underline{u}_{1}, \bar{u}_{1}\right] \times \cdots \times\left[\underline{u}_{n}, \bar{u}_{n}\right]$ for some finite nonnegative $\underline{u}_{i}<\bar{u}_{i}, i=1,2, \ldots, n$. In addition we suppose that $h: X \times U \rightarrow \mathbb{R}$ is a continuously differentiable function denoting current profits. Given a positive discount rate $\rho>0$ and an initial state $x_{0} \in X$, we will discuss the infinite-horizon optimal control problem

$$
\text { (P) : maximize } V(x, u)=\int_{0}^{\infty} \mathrm{e}^{-\rho t} h(x, u) \mathrm{d} t,
$$

\footnotetext{
${ }^{1}$ Note that a slight modification of the proof of the classical maximum principle by Pontryagin et al. (1962) is sufficient for a translation to the infinite-horizon optimal control problem. This has been already noted by Pontryagin et al. (on pp. 189-191) for the special case of a fixed terminal state.

${ }^{2}$ Arrow and Kurz (1970) were aware of this shortcoming (cf. their footnote 1 on p. 46).

${ }^{3}$ Asymptotic stationarity of the (maximized) Hamiltonian was first obtained by Michel (1982) using a different technique.

${ }^{4}$ This and subsequent smoothness assumptions can be somewhat relaxed, but we here deliberately avoid the associated technical difficulties.
} 
subject to (1),

$$
x(0)=x_{0}
$$

and

$$
u \in U \text {. }
$$

We will look for solutions $u$ in the space of bounded measurable functions. Any such function $u$ that satisfies the inclusion (3) for all times $t$ is an admissible control. An admissible pair $(x, u)$ is any admissible control $u$ together with the corresponding state trajectory $x$. Following are the main hypotheses that underlie our conclusions.

H1. The state space $X$ is nonempty, compact and invariant with respect to (1) for all admissible controls $u$ satisfying the inclusion (3).

$\mathrm{H} 2$. There exists $\varepsilon>0$ such that $f_{i} \geqslant \varepsilon$ for all $i=1,2, \ldots, n$.

H3. $\frac{\partial h}{\partial x}>0$.

H4. The function $h$ is strictly concave with respect to $u$.

H5. There exists a $\delta>0$ such that if $u=\left(u_{1}, \ldots, u_{n}\right)^{\prime} \in U$ with $u_{i}=\bar{u}_{i}$ for some $i \in\{1, \ldots, n\}$, then $h(x, u) \leqslant-\delta$. Moreover, for any $x \in X$ there is a $u \in U$ such that $h(x, u) \geqslant 0$.

Assumption $\mathrm{H} 1$ crystallizes the idea that a system that operates using only limited resources stays in a compact set, which is then invariant. This assumption should be naturally satisfied by virtually any real-world economic system, even though many standard models of economic growth ignore saturation effects. ${ }^{5} \mathrm{H} 2$ guarantees a minimum impact of input variations: a finite resource commitment of $u \neq 0$ at the state $x$ adds at least $\varepsilon u$ to the system drift $g(x)$. The monotonicity requirement $\mathrm{H} 3$ ensures the positivity of the adjoint variable. This assumption can be relaxed without affecting the existence of bounds on the adjoint variable (cf. Proposition 2). Note however that positive adjoint variables can be readily interpreted as shadow prices valuing the rate of change at the current state. $\mathrm{H} 4$ ensures the uniqueness of the optimal control $u^{*}$ as a function of $x$ and $\psi$. This assumption is important for the existence of a solution to $(\mathrm{P})$. $\mathrm{H} 5$ requires that there be a negative net return on too large a resource commitment in any one dimension, which is consistent with the notion of decreasing returns to scale (at least up to a positive linear transformation of $h$ ). In particular it imposes that the limits $\bar{u}_{i}$ are large enough for these effects to be noticeable.

Remark 1. A solution $\left(x^{*}, u^{*}\right)$ to $(\mathrm{P})$ does exist. By $\mathrm{H} 1$ the state space $X$ is a compact and invariant set with respect to system (1). Hence, all admissible trajectories are uniformly bounded. In addition, the current value $h$ of the integrand of the objective functional $V$ is bounded from above by

$$
m=\max _{(x, u) \in X \times U} h(x, u)<\infty
$$

\footnotetext{
${ }^{5}$ Note also that the invariance property $\mathrm{H} 1$ does not enter our dynamic optimization problem as a state constraint, since it is required to hold for all admissible state trajectories.
} 
Thus, there exists a nonincreasing positive function $\omega: \mathbb{R}_{+} \rightarrow \mathbb{R}$ such that ${ }^{6}$

$$
\lim _{t \rightarrow \infty} \omega(t)=0
$$

and, for any admissible pair ( $x, u)$ of system (1), subject to (2) and (3), we have

$$
\int_{T}^{\infty} \mathrm{e}^{-\rho t} h(x, u) \mathrm{d} t \leqslant \omega(T)
$$

for all $T>0$. With this and the uniqueness of the optimal control in terms of $(x, \psi)$ (guaranteed by H4), Theorem 3.6 by Balder (1983) implies the existence of a solution to the infinite-horizon optimal control problem $(\mathrm{P}) .^{7}$

\section{Auxiliary constructions}

In this section, we follow the general approach by Aseev et al. (2001b) for the construction of necessary optimality conditions for $(\mathrm{P})$ by considering a sequence of classical optimal control problems $\left(\mathrm{P}_{k}\right)$, each defined on its own finite-time interval $\left[0, T_{k}\right]$. Thereby $T_{k} \leqslant T_{k+1}$ and $T_{k} \rightarrow \infty$ as $k \rightarrow \infty$. We will show that the sequence of optimal controls for $\left(\mathrm{P}_{k}\right)$ converges in $L_{2}$ to the optimal control for (P) entailing strong convergence of the corresponding state trajectories. Our problem $(\mathrm{P})$ thereby does not satisfy the assumptions in Aseev et al. (2001b), so that a number of modifications are necessary. Assume that $\left(x^{*}, u^{*}\right)$ is an optimal pair for the original infinite-horizon optimal control problem (P), and let $\bar{u}=\max \{\|u\|: u \in U\} .{ }^{8}$ Take a sequence of continuously differentiable functions $v_{k}: \mathbb{R}_{+} \rightarrow \mathbb{R}^{m}$, and positive constants $\sigma_{k}<\infty$ (with $\left.\sigma_{k} \leqslant \sigma_{k+1}\right), k=1,2, \ldots$, such that

$$
\begin{aligned}
& \sup _{t \in \mathbb{R}_{+}}\left\|v_{k}(t)\right\| \leqslant 1+\bar{u}, \\
& \int_{0}^{\infty} \mathrm{e}^{-\rho t}\left\|v_{k}-u^{*}\right\|^{2} \mathrm{~d} t \leqslant \frac{1}{k}
\end{aligned}
$$

and

$$
\begin{aligned}
& \omega\left(T_{k}\right) \leqslant \frac{1}{k\left(1+\sigma_{k}\right)}, \\
& \lim _{k \rightarrow \infty} \sigma_{k}=\infty .
\end{aligned}
$$

Such $\left\{v_{k}, \sigma_{k}\right\}, k=1,2, \ldots$, exist and can be obtained using standard approximation methods. Let us now consider the following sequence of classical finite-horizon

\footnotetext{
${ }^{6}$ Without loss of generality we can take $\omega(t)=m \mathrm{e}^{-\rho t} / \rho$.

${ }^{7}$ An appropriate, simple and direct existence proof can also be found in Aseev et al. (2001a).

${ }^{8} \mathrm{We}$ use $\|\cdot\|$ to denote the Euclidean 2-norm unless otherwise indicated.
} 
optimal control problems,

$$
\left(\mathrm{P}_{k}\right): \quad \text { maximize } V_{k}(x, u)=\int_{0}^{T_{k}} \mathrm{e}^{-\rho t}\left(h(x, u)-\frac{\left\|v_{k}-u\right\|^{2}}{1+\sigma_{k}}\right) \mathrm{d} t,
$$

subject to (1), (2), and (3), for $k=1,2, \ldots$. By the Filippov existence theorem (Cesari, 1983, p. 314), there is an optimal control $u_{k}$ solving $\left(\mathrm{P}_{k}\right)$, and we assume that $u_{k}$ and its associated trajectory $x_{k}$ are extended in an arbitrary admissible way onto $\mathbb{R}_{+}$, so that $\left(x_{k}, u_{k}\right)$ forms an admissible pair for $(\mathrm{P})$.

Proposition 1. Let $T>0$. Then $u_{k} \rightarrow u^{*}$ in $L_{2}[0, T]$ as $k \rightarrow \infty$.

Proof. Fix $\bar{\varepsilon}, T>0$ and integer $k_{0}$ such that $T \geqslant T_{k_{0}}$. Then we have for $k \geqslant k_{0}$ :

$$
\begin{aligned}
V_{k}\left(x_{k}, u_{k}\right) & =\int_{0}^{T_{k}} \mathrm{e}^{-\rho t}\left(h\left(x_{k}, u_{k}\right)-\frac{\left\|v_{k}-u_{k}\right\|^{2}}{1+\sigma_{k}}\right) \mathrm{d} t \\
& \leqslant \int_{0}^{T_{k}} \mathrm{e}^{-\rho t} h\left(x_{k}, u_{k}\right) \mathrm{d} t-\frac{\mathrm{e}^{-\rho T}}{1+\sigma_{k}} \int_{0}^{T}\left\|v_{k}-u_{k}\right\|^{2} \mathrm{~d} t .
\end{aligned}
$$

Hence, by optimality of $\left(x_{k}, u_{k}\right)$ for $\left(\mathrm{P}_{k}\right)$ and by optimality of $\left(x^{*}, u^{*}\right)$ for $(\mathrm{P})$ as well as using (5) and (7):

$$
\begin{aligned}
\frac{\mathrm{e}^{-\rho T}}{1+\sigma_{k}} \int_{0}^{T}\left\|v_{k}-u_{k}\right\|^{2} \mathrm{~d} t \leqslant & \int_{0}^{T_{k}} \mathrm{e}^{-\rho t} h\left(x_{k}, u_{k}\right) \mathrm{d} t-V_{k}\left(x^{*}, u^{*}\right) \\
\leqslant & \int_{0}^{T_{k}} \mathrm{e}^{-\rho t} h\left(x_{k}, u_{k}\right) \mathrm{d} t \\
& -\left(V\left(x^{*}, u^{*}\right)-\omega\left(T_{k}\right)-\int_{0}^{\infty} \mathrm{e}^{-\rho t} \frac{\left\|v_{k}-u^{*}\right\|^{2}}{1+\sigma_{k}} \mathrm{~d} t\right) \\
\leqslant & V\left(x_{k}, u_{k}\right)+\omega\left(T_{k}\right)-\left(V\left(x^{*}, u^{*}\right)-\omega\left(T_{k}\right)-\frac{1}{k\left(1+\sigma_{k}\right)}\right) \\
\leqslant & V\left(x_{k}, u_{k}\right)-V\left(x^{*}, u^{*}\right)+\frac{3}{k\left(1+\sigma_{k}\right)} \\
\leqslant & \frac{3}{k\left(1+\sigma_{k}\right)} .
\end{aligned}
$$

Therefore for $k \geqslant \max \left\{k_{0}, 16 \mathrm{e}^{\rho T} / \bar{\varepsilon}^{2}\right\}$ we have using (7):

$$
\left\|u_{k}-u^{*}\right\|_{L_{2}[0, T]} \leqslant\left\|u_{k}-v_{k}\right\|_{L_{2}[0, T]}+\left\|v_{k}-u^{*}\right\|_{L_{2}[0, T]} \leqslant \frac{(1+3) \sqrt{\mathrm{e}^{\rho T}}}{\sqrt{k}} \leqslant \bar{\varepsilon},
$$

which concludes the proof.

As a consequence of Proposition 1 we have for any $T>0$, as $k \rightarrow \infty$ :

$$
\begin{aligned}
& u_{k} \rightarrow u^{*} \text { in } L_{2}[0, T], \\
& x_{k} \rightrightarrows x^{*} \text { on }[0, T], \\
& \dot{x}_{k} \rightarrow \dot{x}^{*} \text { weakly in } L_{1}[0, T] .
\end{aligned}
$$


Without loss of generality (by selecting a subsequence if necessary) we can assume that $u_{k}(t) \stackrel{\text { a.e. }}{\rightarrow} u^{*}(t)$. The strong convergence in $(11)$ is then a consequence of (10), the absolute continuity of $x$ and the boundedness of system equation (1) (see e.g., Filippov, 1988, Theorem 7, p. 12). The weak convergence in (12) is due to the Dunford-Pettis property of $L_{1}$ (Dunford and Schwartz, 1958, IV.8.9, p. 292).

\section{A maximum principle}

We will now formulate a set of necessary conditions that generalize for our class of systems the infinite-horizon maximum principle proved by Pontryagin et al. (1962, pp. 189-191) as well as its later versions mentioned above. First, consider the current-value Hamilton-Pontryagin function

$$
\mathscr{H}\left(x, u, \psi^{0}, \psi\right)=\psi^{0} h(x, u)+\langle\psi, F(x) u+g(x)\rangle,
$$

where $\psi^{0} \geqslant 0$ is a constant, and $\psi(t)$ is the (current-value) adjoint variable for this problem. Whenever $\psi^{0}>0$ it is possible, without loss of generality, to take $\psi^{0}=1$, just by renormalizing the adjoint variable $\psi$. This simplification (to 'normal form') will be rigorously justified in our proof of the necessary optimality conditions below. ${ }^{9}$ Everywhere below it is assumed that $\psi^{0}=1$. The (maximized) Hamiltonian function is defined as

$$
H(x, \psi)=\sup _{u \in U} \mathscr{H}(x, u, \psi)
$$

and straightforward maximization of $\mathscr{H}$ gives $u^{*}(x, \psi)$ as $\mathscr{H}$ is single-peaked by assumption. The next proposition provides necessary optimality conditions for our problem (P), including bounds that imply the transversality condition and asymptotic stationarity of the Hamiltonian obtained by Aseev et al. (2001b).

Proposition 2 (Maximum Principle). Assume that $\mathrm{H} 1$ through $\mathrm{H} 5$ hold. Let $u^{*}$ be an optimal solution of $(\mathrm{P})$, and $x^{*}$ be the corresponding trajectory. Then there exists an absolutely continuous function $\psi: \mathbb{R}_{+} \rightarrow \mathbb{R}^{2}$ such that the following conditions are satisfied:

(i) The function $\psi$ is a solution to the adjoint equation

$$
\dot{\psi}=\rho \psi-\frac{\partial \mathscr{H}\left(u^{*}, x^{*}, \psi\right)}{\partial x} .
$$

(ii) The maximality condition

$$
\mathscr{H}\left(x^{*}(t), u^{*}(t), \psi(t)\right) \stackrel{\text { a.e. }}{=} H\left(x^{*}(t), \psi(t)\right)
$$

holds a.e. on $\mathbb{R}_{+}$.

\footnotetext{
${ }^{9} \mathrm{An}$ example of an infinite-horizon optimal control problem where $\psi^{0}=0$ is optimal has been provided by Halkin (1974).
} 
(iii) The function $\psi(t)$ is strictly positive and bounded on $\mathbb{R}_{+}$. In particular, there exist $\psi, \bar{\psi} \geqslant 0$ such that

$$
\psi \leqslant \psi(t) \leqslant \bar{\psi}
$$

for all $t \in \mathbb{R}_{+}$.

Proof. Consider the sequence of classical optimal control problems $\left(\mathrm{P}_{k}\right)$ constructed above. Let $u_{k}$ be an optimal solution of $\left(\mathrm{P}_{k}\right)$ and $x_{k}$ the corresponding trajectory for $k=1,2, \ldots$. By the Pontryagin maximum principle (Pontryagin et al., 1962) there exists an absolutely continuous function $\psi_{k}=\left(\psi_{1, k}, \ldots, \psi_{n, k}\right)^{\prime}: \mathbb{R}_{+} \rightarrow \mathbb{R}^{n}$ such that the following necessary optimality conditions for $\left(\mathrm{P}_{k}\right)$ hold:

$$
\begin{aligned}
& \dot{\psi}_{k}=\rho \psi_{k}-\frac{\partial \mathscr{H}_{k}}{\partial x}\left(x_{k}, u_{k}, \psi_{k}\right), \\
& \mathscr{H}_{k}\left(x_{k}(t), u_{k}(t), \psi_{k}(t)\right) \stackrel{\text { a.e. }}{=} H_{k}\left(x_{k}(t), \psi_{k}(t)\right), \\
& \psi_{k}\left(T_{k}\right)=0,
\end{aligned}
$$

where we have used the expression $\mathscr{H}_{k}$ for the current-value Hamilton-Pontryagin function in normal form, ${ }^{10}$

$$
\mathscr{H}_{k}\left(x_{k}, u_{k}, \psi_{k}\right)=h\left(x_{k}, u_{k}\right)-\frac{\left\|v_{k}-u_{k}\right\|^{2}}{1+\sigma_{k}}+\left\langle\psi_{k}, F\left(x_{k}\right) u_{k}+g\left(x_{k}\right)\right\rangle
$$

and $H_{k}$ for the corresponding (maximized) Hamiltonian,

$$
H_{k}\left(x_{k}, \psi_{k}\right)=\sup _{u \in U} \mathscr{H}_{k}\left(x_{k}, u, \psi_{k}\right)
$$

with respect to problem $\left(\mathrm{P}_{k}\right)$. We will now concentrate on proving part (iii) of the proposition and first establish the boundedness of the sequence $\left\{\left\|\psi_{k}(0)\right\|\right\}$ for large $k$, which confirms that $\psi^{0}=1$ is an admissible choice. From the boundary condition (19) and adjoint Eq. (17) it is clear that $\psi_{k}(t)$ is strictly positive in $\left[0, T_{k}\right)$. Indeed, using $\mathrm{H} 3$ we have

$$
\dot{\psi}_{i, k}\left(T_{k}\right)=-\frac{\partial h}{\partial x_{i, k}}\left(x_{k}\left(T_{k}\right), u_{k}\left(T_{k}\right)\right)<0
$$

for all $i=1,2, \ldots, n$. Thus, $\psi_{i, k}(t)>0$ in a left-neighborhood of $T_{k}$. This implies that $\psi_{i, k}$ must be strictly positive on all of $\left[0, T_{k}\right)$, since otherwise there would need to exist a $\tau_{i, k} \in\left[0, T_{k}\right)$ such that $\psi_{i, k}\left(\tau_{i, k}\right)=0$ and $\dot{\psi}_{i, k}\left(\tau_{i, k}\right)>0$, which cannot be true due to $\mathrm{H} 3$ and (17). Let $\bar{f}_{x}, \underline{h}_{x}, \underline{h}_{u}$ be defined as in (24)-(26). If $\psi_{i, k}>-\underline{h}_{u} / \varepsilon$, then

\footnotetext{
${ }^{10}$ It is possible to take the normal form (i.e., $\left.\psi_{k}^{0}=1\right)$ without loss of generality, since $\left(\mathrm{P}_{k}\right)$ is a problem on a fixed time interval $\left[0, T_{k}\right]$ with free terminal state, and thus $\psi_{k}^{0} \neq 0$.
} 
(using $\mathrm{H} 2$ and the definition of $\underline{h}_{u}$ )

$$
\begin{aligned}
\frac{\partial \mathscr{H}_{k}}{\partial u_{k}}\left(x_{k}, u_{k}, \psi_{k}\right) & =\frac{\partial h}{\partial u_{k}}\left(x_{k}, u_{k}\right)+\frac{2\left\|v_{k}-u_{k}\right\|}{1+\sigma_{k}}+\left\langle\psi_{k}, F\left(x_{k}\right)\right\rangle \\
& \geqslant \underline{h}_{u}+\varepsilon \sum_{i=1}^{n} \psi_{i, k}>0
\end{aligned}
$$

which implies $u_{i, k}=\bar{u}_{i}$. Let us consider the longest time interval $\left[0, \bar{T}_{k}\right], 0 \leqslant \bar{T}_{k} \leqslant T_{k}$, on which at least one component of the adjoint variable $\psi_{k}$ is larger than some critical lower bound, ${ }^{11}$

$$
\psi_{i, k}>-\underline{h}_{u} / \varepsilon .
$$

Relation (21) implies as shown in (20) that the $i$ th component of the optimal control $u_{k}$ must be maximal, i.e., $u_{i, k}=\bar{u}_{i}$. This in turn implies by $\mathrm{H} 5$ that $h \leqslant-\delta$, which means that there is a finite negative contribution to the objective function of $-\delta$ multiplied by the length of the time interval for which (21) is satisfied. ${ }^{12}$ Clearly, due to $\mathrm{H} 5$, the optimal profit cannot be negative and

$$
0 \leqslant \int_{0}^{T_{k}} \mathrm{e}^{-\rho t} h\left(x_{k}, u_{k}\right) \mathrm{d} t \leqslant-\frac{\delta}{\rho}\left(1-\mathrm{e}^{-\rho \bar{T}_{k}}\right)+\omega\left(T_{k}\right),
$$

whence with $\omega\left(T_{k}\right)=m \mathrm{e}^{-\rho T_{k}} / \rho$ and $T_{k} \rightarrow \infty$ we obtain that the $\bar{T}_{k}$ 's are uniformly bounded, $\bar{T}_{k} \leqslant T^{\prime}$, with

$$
T^{\prime}=\frac{1}{\rho} \ln \left(1+\frac{m}{\delta}\right)
$$

Thus, there is a bounded sequence ${ }^{13} \tau_{k} \in\left[0, T^{\prime}\right], k=1,2, \ldots$, such that $\psi_{i, k}\left(\tau_{k}\right) \leqslant$ $-\underline{h}_{u} / \varepsilon$ for all $i=1,2, \ldots, n$. By (17) and (24) and (25)

$$
\dot{\psi}_{i, k} \geqslant \rho \psi_{i, k}-\underline{h}_{x}-\bar{f}_{x} \sum_{j=1}^{n} \psi_{j, k}
$$

holds on $\left[0, T_{k}\right]$, so that after solving this differential inequality with boundary condition $\psi_{i, k}\left(T^{\prime}\right) \leqslant-\underline{h}_{u} / \varepsilon$, we have that

$$
\psi_{i, k}(t) \leqslant \frac{\underline{h}_{x}}{n \bar{f}_{x}-\rho}\left(\mathrm{e}^{\left(n \bar{f}_{x}-\rho\right)\left(T^{\prime}-t\right)}-1\right)-\frac{\underline{h}_{u}}{\varepsilon} \mathrm{e}^{\left(n \bar{f}_{x}-\rho\right)\left(T^{\prime}-t\right)}
$$

\footnotetext{
${ }^{11}$ The lower bound is independent of $k$. There may be more than one interval on which (21) holds, but we are only interested in the longest, the length of which is defined as the supremum of the lengths of all such intervals.

${ }^{12}$ Due to the principle of optimality (Bellman, 1957, p. 83) we can take, without loss of generality, the beginning of the time interval to be zero. Otherwise just consider a new problem of type (P) with the initial state equal to the state at the time when $\psi_{i, k} \geqslant-\underline{h}_{u} / \varepsilon$ in the original problem.

${ }^{13}$ Of course $\tau_{k}<T_{k}$ since $\psi_{i, k}\left(T_{k}\right)=0$ by (19), but this is immaterial to the discussion, since the sequence of $T_{k}$ 's, $k=1,2, \ldots$, is unbounded.
} 
for all $t \in\left[0, \min \left\{T_{k}, T^{\prime}\right\}\right]$ as long as $n \bar{f}_{x}>\rho .{ }^{14}$ Hence with (22)

$$
\left\|\psi_{k}(0)\right\| \leqslant \sqrt{n}\left[\frac{\underline{h}_{x}}{n \bar{f}_{x}-\rho}\left(\left(1+\frac{m}{\delta}\right)^{\left(n \bar{f}_{x} / \rho\right)-1}-1\right)-\frac{\underline{h}_{u}}{\varepsilon}\left(1+\frac{m}{\delta}\right)^{\left(n \bar{f}_{x} / \rho\right)-1}\right],
$$

i.e., $\left\|\psi_{k}(0)\right\|$ is uniformly bounded (independent of $k$ ). Consider now the increasing sequence of time intervals $I_{j}=\left[0, T_{j}\right], j=1,2, \ldots$, and on each $I_{j}$ consider the sequences $\left\{u_{k}\right\},\left\{x_{k}\right\}$, and $\left\{\psi_{k}\right\}$. Given $I_{j}$, the sequence $\left\{\left\|\psi_{k}(0)\right\|\right\}$ is uniformly bounded. By virtue of the Bellman-Gronwall Lemma (Sontag, 1998, pp. 474-475) and the adjoint equation (17) one can assume, without loss of generality, that there exists an absolutely continuous function $\psi_{k}: \mathbb{R}_{+} \rightarrow \mathbb{R}^{n}$ such that (as $\left.k \rightarrow \infty\right)$

$$
\begin{aligned}
& \psi_{k} \rightrightarrows \psi \quad \text { on } I_{j}, \\
& \dot{\psi}_{k} \rightarrow \dot{\psi} \quad \text { weakly in } L_{1}\left(I_{j}\right) .
\end{aligned}
$$

Given the sequence $\left\{I_{j}\right\}$ we can pass on each $I_{j}, j=1,2, \ldots$, from the sequence $\left\{\psi_{k}\right\}$ to a subsequence converging to $\psi$. By selecting a diagonal subsequence, there is an absolutely continuous function $\psi: \mathbb{R}_{+} \rightarrow \mathbb{R}$ such that, for any $T>0$, we have (as $k \rightarrow \infty)$ :

$$
\begin{aligned}
& \psi_{k} \rightrightarrows \psi \quad \text { on }[0, T], \\
& \dot{\psi}_{k} \rightarrow \dot{\psi} \quad \text { weakly in } L_{1}[0, T] .
\end{aligned}
$$

By Proposition 1 and the discussion thereafter, we have that $u_{k} \rightarrow u^{*}$ strongly in $L_{2}[0, T]$ as $k \rightarrow \infty$, and $x_{k} \rightrightarrows x^{*}$ uniformly on $[0, T]$ as $k \rightarrow \infty$. Using the Mazur theorem (e.g., Mordukhovich, 1988), the absolutely continuous function $\psi$ is a solution to the adjoint Eq. (17) on any time interval $[0, T], T>0$. This proves part (i).

The maximality condition (15) follows from passing to the limit in (18). This proves part (ii).

The strict positivity of $\psi$ is a direct consequence of $\psi_{i, k}(t)>0$ on $\left[0, T_{k}\right)$, for $k \rightarrow \infty$. Since $T^{\prime}$ is the maximum length of any time-interval in which $\psi_{i, k} \geqslant-\underline{h}_{u} / \varepsilon$, our upper bound for $\left\|\psi_{k}(0)\right\|$ is also a uniform upper bound of $\left\|\psi_{k}(t)\right\|$ on $\left[0, T_{k}\right]$ for all $k=1,2, \ldots$, which implies (23) in Corollary 1 for $k \rightarrow \infty$. Thus we have shown part (iii), which concludes the proof.

Corollary 1. Some a priori estimates for the bounds on the adjoint variable are given by $\underline{\psi}=0$ and $\bar{\psi}=\left(\bar{\psi}_{1}, \ldots, \bar{\psi}_{n}\right)^{\prime}$ with

$$
\bar{\psi}_{i}=\frac{\underline{h}_{x}}{n \bar{f}_{x}-\rho}\left(\left(1+\frac{m}{\delta}\right)^{\left(n \bar{f}_{x} / \rho\right)-1}-1\right)-\frac{\underline{h}_{u}}{\varepsilon}\left(1+\frac{m}{\delta}\right)^{\left(n \bar{f}_{x} / \rho\right)-1}
$$

for $i=1,2, \ldots, n$, provided that $n \bar{f}_{x}>\rho .{ }^{15}$ Thereby we have set

$$
\bar{f}_{x}=\max _{(x, u) \in X \times U}\left\{\frac{\rho}{n},\left\|\frac{\partial(F(x) u+g(x))}{\partial x}\right\|_{\infty}\right\},
$$

\footnotetext{
${ }^{14}$ For $n \bar{f}_{x}=\rho$ it is $\psi_{i, k} \leqslant \underline{h}_{x}\left(T^{\prime}-t\right)-\underline{h}_{u} / \varepsilon$ for all $t \in\left[0, \min \left\{T_{k}, T^{\prime}\right\}\right]$.

${ }^{15}$ In the (degenerate) case when $n \bar{f}_{x}=\rho$, we obtain $\bar{\psi}_{i}=\left(\underline{h}_{x} / \rho\right) \ln (1+m / \delta)-\underline{h}_{u} / \varepsilon$.
} 


$$
\begin{aligned}
& \underline{h}_{x}=\min _{\substack{(x, u) \in X \times U \\
1 \leqslant i \leqslant n}}\left\{\frac{\partial h(x, u)}{\partial x_{i}}\right\}>0, \\
& \underline{h}_{u}=\min _{\substack{(x, u) \in X \times U \\
1 \leqslant i \leqslant n}}\left\{\frac{\partial h(x, u)}{\partial u_{i}}\right\}<0 .
\end{aligned}
$$

A proof of the last assertion is a byproduct of the proof of Proposition 2. The strict positivity of the adjoint variable in (iii) is essentially a consequence of $\mathrm{H} 3$. Note that 'reversing' hypothesis $\mathrm{H} 3$ for some or all components (i.e., $\partial h / \partial x_{i}<0$ for some $i \in\{1, \ldots, n\})$ will render the corresponding components of $\psi$ strictly negative (i.e., $\left.\psi_{i}<0\right)$. The existence of a lower bound $\psi$ (possibly negative) is independent of $\mathrm{H} 3$ and can be shown in analogy to the proof of the existence of an upper bound $\bar{\psi}$.

Remark 2. The state trajectory is bounded, i.e., $x^{*}(t) \in X$ for all $t \in \mathbb{R}_{+}$. Proposition 2 provides growth conditions in the form of inequalities, stronger that the 'natural' transversality condition ${ }^{16}$

$$
\lim _{t \rightarrow \infty} \mathrm{e}^{-\rho t} \psi(t)=0,
$$

proposed for instance by Arrow and Kurz (1970), which is a direct consequence of (16). Thus, condition (16) together with the boundedness of $h$ on $X \times U$ implies the asymptotic stationarity of the Hamiltonian,

$$
\lim _{t \rightarrow \infty} \mathrm{e}^{-\rho t} H\left(x^{*}(t), \psi(t)\right)=0 .
$$

Remark 3. From (14) and (15) we get that

$$
\begin{aligned}
\frac{\mathrm{d}}{\mathrm{d} t}\left(\mathrm{e}^{-\rho t} H\left(x^{*}(t), \mathrm{e}^{\rho t} \psi(t)\right)\right) & \stackrel{\text { a.e. }}{=} \frac{\partial}{\partial t}\left(\mathrm{e}^{-\rho t} \mathscr{H}\left(x^{*}(t), u^{*}(t), \mathrm{e}^{\rho t} \psi(t)\right)\right) \\
& =-\rho \mathrm{e}^{-\rho t} h\left(x^{*}(t), u^{*}(t)\right)
\end{aligned}
$$

which, integrated over $\mathbb{R}_{+}$, together with (28) yields

$$
H\left(0, x_{0}, \psi_{0}\right)=\rho \int_{0}^{\infty} \mathrm{e}^{-\rho t} h\left(x^{*}, u^{*}\right) \mathrm{d} t=\rho V\left(x^{*}, u^{*}\right) .
$$

Thus, we have obtained an expression for $V^{*}:=V\left(x^{*}, u^{*}\right)$ in terms of the initial conditions $x_{0}, \psi_{0}$ :

$$
V^{*}=\frac{1}{\rho} \max _{u \in U}\left\{h\left(x_{0}, u\right)+\left\langle\psi_{0}, F\left(x_{0}\right) u+g\left(x_{0}\right)\right\rangle\right\} .
$$

\section{Example}

We now give a concrete example for the application of our result, based on a classic model for optimal advertising spending by Vidale and Wolfe (1957). Consider

\footnotetext{
${ }^{16}$ Such a transversality condition need not hold, even for simple infinite-horizon optimal control problems. An appropriate counterexample was given by Halkin (1974).
} 
the system

$$
\dot{x}=(1-x) a^{\kappa}-\beta x
$$

with some constants $\beta>0$ and $\kappa \in(0,1)$. The control variable $a$ denotes the amount of advertising and the state variable $x$ the installed base, normalized to the interval $[0,1]$. The problem is, given an initial state $x(0)=x_{0}$, to maximize discounted profits $^{17}$

$$
\Pi(x, a)=\int_{0}^{\infty} \mathrm{e}^{-\rho t}\left((1-x) a^{\kappa}-c a\right) \mathrm{d} t,
$$

where $c>0$ is the cost of advertising. Note first that after a simple integration by parts using (30) we have $\Pi(x, a)=-x_{0}+c V(x, u)$, where $u=a^{\kappa}$ and

$$
V(x, u)=\int_{0}^{\infty} \mathrm{e}^{-\rho t}\left(\gamma x-u^{1 / \kappa}\right) \mathrm{d} t
$$

with $\gamma=(\beta+\rho) / c$. Let $F(x)=1-x, g(x)=-\beta, h(x, u)=\gamma x-u^{1 / \kappa}$ and furthermore $U=[0, \bar{u}]$ and $X=[0, \bar{x}]$, where $\bar{x}=\bar{u} /(\beta+\bar{u})<1$. Thus $\mathrm{H} 1$ is satisfied and so is $\mathrm{H} 2$ for $\varepsilon=1-\bar{x}$. H3 and H4 can be directly verified and do hold. H5 is satisfied for any $\bar{u} \geqslant \gamma^{\kappa}$ with $\delta=\bar{u}^{1 / \kappa}-\gamma \bar{x}>0$. Given $x_{0} \in X$ we can therefore use the necessary optimality conditions for $(\mathrm{P})$ in Proposition $2 .{ }^{18}$ By the positivity of the adjoint variable we find

$$
u^{*}=\min \left\{\bar{u},(\kappa(1-x) \psi)^{\kappa /(1-\kappa)}\right\}>0
$$

and for $\bar{u}>\kappa \bar{\psi}$ we obtain the Hamiltonian system ${ }^{19}$

$$
\begin{aligned}
& \dot{x}=(1-x)^{1 /(1-\kappa)}(\kappa \psi)^{\kappa /(1-\kappa)}-\beta x, \\
& \dot{\psi}=-\gamma+(\rho+\beta) \psi+(\kappa(1-x))^{\kappa /(1-\kappa)} \psi^{1 /(1-\kappa)} .
\end{aligned}
$$

This system possesses a turnpike state $\left(\hat{x}^{*}, \hat{\psi}^{*}\right)$ characterized by $\left.(\dot{x}, \dot{\psi})\right|_{\left(\hat{x}^{*}, \hat{\psi}^{*}\right)}=0$, or equivalently

$$
\begin{aligned}
& \kappa \gamma=\left(\frac{\rho+\beta}{\left(1-\hat{x}^{*}\right)^{1 / \kappa}}+\frac{\beta \hat{x}^{*}}{\left(1-\hat{x}^{*}\right)^{\left(1-\kappa^{2}\right) /(\kappa(1-\kappa))}}\right)\left(\beta \hat{x}^{*}\right)^{(1-\kappa) / \kappa}, \\
& \hat{\psi}^{*}=\frac{\left(\beta \hat{x}^{*}\right)^{(1-\kappa) / \kappa}}{\kappa\left(1-\hat{x}^{*}\right)^{1 / \kappa}} .
\end{aligned}
$$

For $\bar{u}$ large enough there is a (unique) turnpike; a corresponding qualitative phase portrait is shown in Fig. 1. From there it is clear that the only trajectories satisfying

\footnotetext{
${ }^{17}$ The demand is equal to the positive inflow to the installed base, $(1-x) a^{\kappa}$. The firm is assumed to be a price taker in a market for durable goods, where the price has been normalized to 1 . The products have a characteristic lifetime of $1 / \beta$ before being discarded. The exponent $\kappa$ models the effect of decreasing returns to investment in advertising.

${ }^{18}$ Relations (24)-(26) yield $\bar{f}_{x}=\max \{\rho, \beta+\bar{u}\}, \underline{h}_{x}=\gamma$, and $\underline{h}_{u}=-1$.

${ }^{19}$ This may seem circular as $\bar{u}$ is used to compute $\bar{\psi}$. However, it is possible to iteratively set $\bar{u}$ to a lower value and determine the corresponding a priori bound $\bar{\psi}$ which can then be used to correct the choice for $\bar{u}$ upwards.
} 


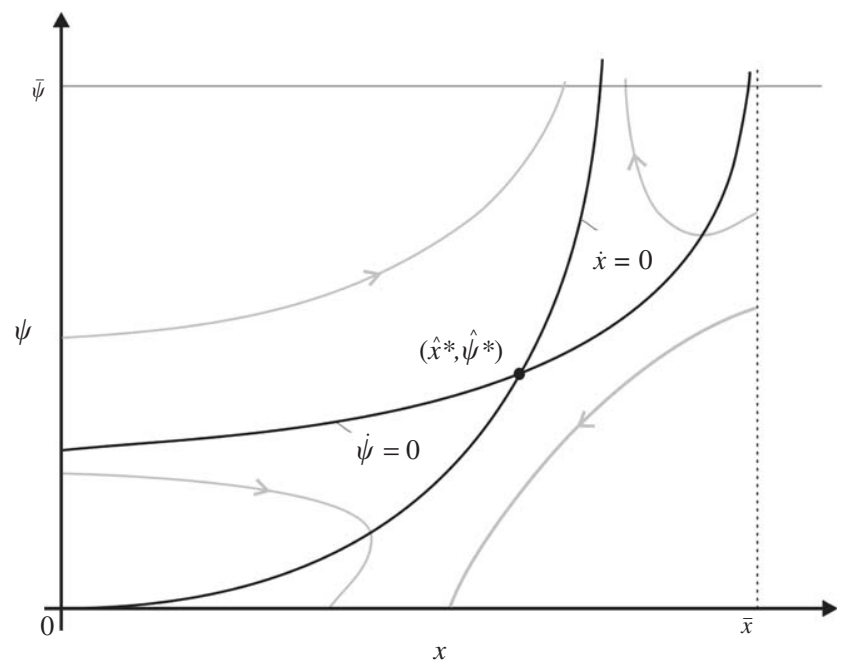

Fig. 1. Phase portrait of the Hamiltonian system (31) and (32) for $(x, \psi) \in X \times(0, \bar{\psi}]$.

the bounds on the adjoint variable $\psi$ are the ones which asymptotically converge to the turnpike. All other trajectories of the Hamiltonian system do not satisfy the bounds on the adjoint variable. We thus remark (without detailed proof) that uniqueness, asymptotic convergence, and the precise form of the optimal solution to our example problem of form (P) follow in essence directly from the necessary optimality conditions in Proposition 2.

\section{Discussion}

We have provided an extended set of necessary optimality conditions for a general class of infinite-horizon dynamic optimization problems. The class of relevant problems satisfies natural invariance and saturation assumptions, thereby encompassing a broad range of economic problems. The maximum principle formulated here includes bounds on the (current-value) adjoint variables, which imply asymptotic stationarity of the (maximized) Hamiltonian and the 'natural' transversality condition in Arrow-Kurz form. We have also given some a priori estimates for these bounds, which generally can be improved upon through more careful componentwise considerations and by using the structure of a particular problem. The bounds on the adjoint variable can be used to generate a grid of initial conditions of the adjoint variable for an approximation of the optimal solution of $(\mathrm{P})$ by forward simulation of the Hamiltonian system or they may be useful as an intermediate theoretical result, for instance on the way to establishing asymptotic convergence of the optimal trajectory (cf. the example discussed above). 


\section{Acknowledgements}

This research was supported by a David Morgenthaler Faculty Scholar Award at Stanford University, by the Risk Management and Decision Processes Center at the Wharton School of the University of Pennsylvania, and by the International Institute for Applied Systems Analysis, Austria. I would like to thank Sergei Aseev for numerous fruitful discussions and Paul Kleindorfer for his encouragement throughout the work on this project. Of course, any remaining errors are the sole responsibility of the author.

\section{References}

Arrow, K.J., Kurz, M., 1970. Public Investment, The Rate of Return, and Optimal Fiscal Policy. Johns Hopkins Press, Baltimore.

Aseev, S., 1999. Methods of regularization in nonsmooth problems of dynamic optimization. Journal of Mathematical Sciences 94 (3), 1366-1393.

Aseev, S., Kryazhimskii, A., Tarasyev, A., 2001a. First order necessary optimality conditions for a class of infinite horizon optimal control problems. Technical Report IR-01-007, International Institute for Applied Systems Analysis, Laxemburg, Austria.

Aseev, S., Kryazhimskii, A., Tarasyev, A., 2001b. The Pontryagin maximum principle and transversality conditions for an optimal control problem with infinite time interval. Proceedings of the Steklov Institute of Mathematics, Moscow, vol. 233, pp. 64-80.

Balder, E., 1983. An existence result for optimal economic growth problems. Journal of Mathematical Analysis and Applications 95 (1), 195-213.

Bellman, R.E., 1957. Dynamic Programming. Princeton University Press, Princeton, NJ.

Cesari, L., 1983. Optimization: Theory and Applications. Springer, New York.

Dunford, N., Schwartz, J., 1958. Linear Operators, Part I: General Theory. Wiley Interscience, New York.

Filippov, A., 1988. Differential Equations with Discontinuous Righthand Sides. Kluwer Academic Publishers, Boston.

Halkin, H., 1974. Necessary conditions for optimal control problems with infinite horizons. Econometrica 42 (2), 267-272.

Lee, E.B., Markus, L., 1967. Foundations of Optimal Control Theory. Wiley, New York.

Michel, P., 1982. On the transversality condition in infinite horizon optimal problems. Econometrica 50 (4), 975-985.

Mordukhovich, B., 1988. Approximation Methods in Problems of Optimization and Control. Nauka, Moscow (in Russian).

Pontryagin, L.S., Boltyanskii, V.G., Gamkrelidze, R.V., Mishchenko, E.F., 1962. The Mathematical Theory of Optimal Processes. Wiley Interscience, New York.

Sontag, E.D., 1998. Mathematical Control Theory: Deterministic Finite Dimensional Systems. Springer, New York.

Vidale, M., Wolfe, H., 1957. An operations research study of sales response to advertising. Operations Research 5 (3), 370-381.

Weber, T.A., 2001. Can optimal receding-horizon decision making be unstable? Working Paper No. 01-1002, Department of Operations and Information Management, Wharton School, University of Pennsylvania. 